\title{
Uso de videos educativos en la materia de programación durante la etapa básica de ingeniería
}

\author{
Araceli C. Justo-López, Wendolyn E. Aguilar-Salinas, Maximiliano de las Fuentes-Lara, María A. Astorga-Vargas \\ Universidad Autónoma de Baja California, Blvd. Benito Juárez y Calle de la Normal S/N, Colonia Insurgentes Este, \\ Mexicali, Baja California, CP: 21280, México. (correo-e: araceli.justo@uabc.edu.mx, aguilar.wendolyn@uabc.edu.mx, \\ maximilianofuentes@uabc.edu.mx, angelicaastorga@uabc.edu.mx)
}

Recibido Mar. 1, 2021; Aceptado May. 3, 2021; Versión final Jun. 27, 2021, Publicado Dic. 2021

\begin{abstract}
Resumen
El objetivo de este estudio es evaluar una estrategia didáctica de videos educativos implementados para mejorar los resultados de los alumnos en la materia de programación en carreras de ingeniería. La muestra fue conformada por un total de 55 estudiantes de segundo semestre de ingeniería durante el ciclo escolar 2019-1 (febrero a junio) de la Universidad Autónoma de Baja California (UABC), México. Se recolectan, describen y analizan las percepciones de los estudiantes sobre el uso de videos educativos y el impacto en su aprovechamiento académico. Los resultados muestran que los videos educativos son una herramienta de apoyo adicional que motiva a los estudiantes a aprender programación. Adicionalmente, se observa un incremento del $13.47 \%$ en el porcentaje de aprobación en las evaluaciones. Se concluye que el uso de videos educativos es considerado aceptable, como una herramienta valiosa de apoyo, por los estudiantes, aunque para ellos es importante contar con la guía y asesoría del docente.
\end{abstract}

Palabras clave: educación superior; estrategia didáctica; ingeniería; programación; videos educativos

\section{Use of educational videos in a programming course at the initial stages of engineering degrees}

\begin{abstract}
The primary objective of this research study is to assess a didactic strategy implemented to improve learning of engineering students in a programing course. The sample consists of 55 undergraduate second-semester engineering students enrolled in the year 2019 (February-June) at the Universidad Autónoma de Baja California (UABC), Mexico. Student perceptions about the use of educational videos and their impact on academic results are collected, described, and analyzed. The results show that educational videos are tools that encourage students to learn programming. In addition, there is an increase of $13.47 \%$ in approval rate for in-class evaluations. It is concluded that the use of educational videos is considered acceptable as a valuable support tool by students, but they consider that it is important to also have the guide and assistance from professors.
\end{abstract}

Keywords: higher education; teaching strategy; engineering; programming; educational videos 


\section{INTRODUCCIÓN}

La programación es una asignatura que se orienta al desarrollo de habilidades para la solución de problemas aplicables al mundo real, resaltando la creatividad, la innovación, el pensamiento lógico y crítico (Fracchia et al., 2016). Bashir y Hoque (2016), expresan que aprender a programar es difícil ya que requiere la comprensión de los antecedentes teóricos, el uso práctico de la semántica y la codificación sintáctica y las habilidades algorítmicas. El estudiante de programación debe desarrollar la capacidad de crear algoritmos lógicamente, lo que involucra habilidades del pensamiento e imaginación (Hubalovsky, 2015). En Bosse y Gerosa (2017) se expone la importancia de enseñar análisis y diseño antes que programación, y la limitación que representa el concentrar esfuerzos para la detección de errores de sintaxis en lugar de comprender la lógica de la programación.

Otro de los factores que influyen en la dificultad del aprendizaje de la programación es la carga cognitiva de los materiales de aprendizaje, los cuales contienen varios textos y representaciones visuales para aprender conceptos de programación, incluyendo estructuras de control de flujos, como ciclos y sentencias IF-ELSE, sintaxis y códigos de programación, palabras claves, diagramas de flujo, algoritmos y pseudocodigos, con la desventaja de que en una sola clase se abordan varios de estos conceptos (Matthews et al., 2015). Además, la programación es difícil por la construcción de los modelos mentales necesarios para el desarrollo de solución de problemas. Es por esto que diversas investigaciones han intentado hacer que la programación de computadoras sea divertida, que motive a los estudiantes para aumentar su rendimiento (Khaleel, Shaari y Wook, 2019).

El siglo XXI demanda graduados universitarios con pensamiento computacional y habilidades de programación de computadoras, aunque pertenezcan a disciplinas diferentes. Es por esto que el conocimiento básico de programación forma parte de casi todos los programas de ingeniería, puesto que requiere de habilidades de pensamiento crítico, resolución de problemas, pensamiento computacional y nuevos diseños de sistemas (Topalli y Cagiltay, 2018). En Derus y Ali (2016) se expresa que el surgimiento de la tecnología computacional en la era del Internet y la globalización, ha hecho que el aprendizaje de la programación sea esencial, puesto que no solo se requiere en el campo de la informática, sino que también en otras áreas como las ciencias, matemáticas e ingeniería. En países como Reino Unido, Estonia y España, los estudios de programación se implementan como obligatorios en la educación primaria para niños desde los cinco años (Kanoh, 2018). Armoni et al., (2015) explican que el aprendizaje de las ciencias de la computación en escuelas intermedias ayuda en la mejora del aprendizaje, la eficiencia de la enseñanza y los factores afectivos.

Los estudiantes pueden perder la motivación y el interés después del primer fallo en la codificación, pero los instructores deben estar preparados para ayudar y tratar de evitar esta situación. Ha sido labor de los docentes sensibilizarlos sobre la importancia de la programación en el área de ingeniería y en general en la vida de un profesional, como una herramienta para la solución de problemas. Se considera esencial incorporar en las aulas estrategias que despierten ese interés en el alumno y sensibilizar de la importancia de la materia en el área de ingeniería. De acuerdo con Odo y Odo (2016), el interés tiene un papel preponderante en el patrón de aprendizaje individual, ya que puede garantizar la atención, alentar y apoyar el estilo de aprendizaje del estudiante. El maestro debe involucrar a los estudiantes en entornos de aprendizaje empíricos para que puedan experimentar el aprendizaje con elementos que disfruten.

Internet ofrece múltiples herramientas tecnológicas gratuitas que pueden ser de utilidad en el ámbito educativo, mientras se utilicen bajo una instrucción adecuada que asegure la construcción de aprendizajes. En este sentido, se identifica a los recursos audiovisuales como materiales idóneos, a los que los estudiantes pueden tener acceso con facilidad dentro y fuera del aula; específicamente, los videos educativos desarrollados por docentes resultan de gran apoyo, puesto que permiten que se evoquen las explicaciones, el acompañamiento y los estímulos de un docente las veces que sean necesarias. La visualización de un programa es una herramienta de soporte al aprendizaje de la programación que puede ayudar a la comprensión de los procesos implicados ya que los humanos tienen mejores capacidades para procesar información visual (Topalli y Cagiltay, 2018).

La unidad de aprendizaje de Programación, ofertada en la Facultad de Ingeniería (FIM) de la Universidad Autónoma de Baja California (UABC), se ubica en el segundo semestre de la etapa básica de formación del ingeniero, una etapa en la cual los estudiantes aún no tienen definida la rama de la ingeniería en la que desean especializarse, puesto que se encuentran inscritos en un tronco común que dura dos semestres. Por tanto, los estudiantes que cursan esta unidad de aprendizaje tienen diferentes perfiles e intereses hacia el estudio de la programación: algunos tienen conocimientos previos de bachillerato, mientras que otros nunca han visto temas relacionados en sus etapas escolares anteriores. Los estudiantes manifiestan poco interés en la materia a pesar de ser obligatoria, ya que no visualizan cómo puede serles de utilidad para las áreas de la ingeniería que no tienen una relación directa con el desarrollo de software. Esta situación ha llevado a la asignatura a tener un alto índice de reprobación. 
A fin de aprovechar el acceso que los estudiantes tienen a internet y su gusto por ver videos de diferentes índoles, en este trabajo se presentan los resultados de aplicar como estrategia didáctica un conjunto de videos educativos sobre programación en lenguaje $\mathrm{C}$, enfocados en temas de mayor dificultad para los estudiantes, como lo son: estructuras repetitivas, arreglos unidimensionales y arreglos bidimensionales. Los videos fueron utilizados como una herramienta de apoyo en la enseñanza y aprendizaje de la Programación, para evaluar su impacto en el rendimiento de los estudiantes.

\section{OTROS ANTECEDENTES}

El fundamento teórico se presenta en dos secciones que describen los rasgos que debe cumplir la estrategia didáctica, como lo son: características y su uso en la educación superior.

\section{El video educativo y sus características}

Se define al video educativo como un material audiovisual que es de utilidad en el proceso de enseñanza y aprendizaje por su función motivadora que contribuye al aprendizaje, y que en el ámbito escolar cumple funciones informativas, motivadoras y expresivas (Eslava, López, Lloclla y Vidaurre, 2018).

Se definen cinco funciones básicas que puede desempeñar un video, dependiendo de cómo decida implementarse dentro del proceso educativo (Ríos, 2011). Puede funcionar para: despertar el interés sobre una temática particular, ya que su finalidad principal es presentarle al receptor un contenido y promover una actitud de apertura para conocer; introducir un tema, brindando una visión general del mismo en donde se destacan conceptos clave que sirvan de anclaje cognitivo; desarrollar un tema, en la medida en que se utilice como apoyo para la intervención del docente; confrontar ideas, apelando al análisis y el diálogo, y cerrar un tema, cuando se utiliza para corroborar la construcción de aprendizajes por parte del estudiante.

Tapia et al., (2020) indican que los videos académicos motivan a los estudiantes a aprender sin ninguna presión, estimulando su pensamiento y al mismo tiempo les permiten resolver sus problemas sobre la materia del curso. En concordancia con estas funciones, se retoman las propuestas de García (2014), quien describe que el video educativo posee el potencial de convertirse en: a) instrumento de formación para el estudiante, ya que supone una herramienta de apoyo para el desarrollo de las competencias establecidas por el currículum; b) medio de perfeccionamiento del profesorado en sus conocimientos profesionales y en sus destrezas didácticas, en el sentido de que permite al docente que lo elabora desarrollar su capacidad para impartir un conocimiento en un tiempo reducido, así como observar y modificar su actuación para mejorar su desempeño; c) herramienta de investigación didáctica, debido a que su uso puede brindar datos relevantes en torno a la interacción que existe entre el docente, el alumno y el contenido, además de información sobre los procesos cognitivos en ambientes de aprendizaje apoyados por tecnologías.

\section{El uso de videos en la educación superior}

Una de las mayores ventajas de los videos educativos en la educación superior es que se tratan de recursos ampliamente difundidos por los jóvenes, los cuales no solo cuentan con la capacidad de descargarlos para uso personal, sino que también pueden crear páginas para difundirlos (Solano y Sánchez, 2010). Es evidente la preferencia que los estudiantes de hoy en día manifiestan sobre su uso, por lo que son considerados como una alternativa viable para implementar estrategias de aprendizaje atractivas y prácticas, diferentes a la tradicionales, que capten la atención de los estudiantes nativos digitales, favorezcan su motivación y apoyen en su aprendizaje (Lozano et al., 2018).

Para el docente, la inclusión permanente de recursos audiovisuales se traduce en dos posibilidades: primero, de adaptarse a los cambios provocados por la evolución de la tecnología, y después de acceder a una oferta más amplia de herramientas para la formación del estudiante (De la Fuente et al., 2018). Es fundamental tener en claro que la innovación en el proceso de enseñanza-aprendizaje "no se encuentra solo en el uso mayoritario o no de estos recursos sino también en el sentido didáctico y pedagógico que en torno y a través de estas herramientas se ofrezca" (Trujillo, 2011, p. 64). Cabe mencionar que este medio de aprendizaje puede no ser el más adecuado para todos los alumnos, y que algunos alumnos se vean más favorecidos que otros a partir de su implementación debido a los diferentes estilos de aprendizaje de cada uno de ellos (Ann y Carr, 2017).

Para lograr resultados efectivos, no basta simplemente con introducir al video en el contexto educativo, ya que este debe ser incluido como un elemento interactivo de la planeación, la ejecución y la evaluación del proceso. En un curso apoyado por tecnología, el diseño instruccional describe el curso, los objetivos de aprendizaje, el temario y la metodología específica de cada estrategia de aprendizaje; indica las responsabilidades tanto del docente como de cada alumno y, por supuesto, el sistema de evaluación, autoevaluación y metacognición (Vázquez et al., 2013). 


\section{METODOLOGÍA}

La metodología se presenta en cinco subsecciones en las que se describe su elaboración, diseño e implementación de la estrategia didáctica, los sujetos de estudio, los instrumentos utilizados y la evaluación de la estrategia.

\section{Desarrollo de videos educativos}

Los videos educativos se desarrollaron considerando la metodología propuesta por Gago y Vico (2013), donde se sugieren las siguientes etapas para el desarrollo de material audiovisual: a) Fijar los objetivos del video, b) Buscar información y recursos visuales ligados a los objetivos, c) Generar un guion preciso y concreto del video, d) Generar contenido propio audiovisual, e) Editar el video, f) Cerrar y publicar el video en la red.

Los videos tuvieron duración de 10 a 20 minutos y cada uno siguió un objetivo específico en relación con el tema que mostraba. Su estructura se ajustó a una rúbrica diseñada con base en las recomendaciones de Cano (2015) retomando algunos de sus indicadores. La rúbrica contiene datos de identificación y el apartado de evaluación. En el apartado de evaluación se considera el diseño y producción audiovisual, donde se revisan aspectos relativos a la presentación de datos dentro del vídeo (universidad, facultad, logos institucionales, temática y créditos), así como a la calidad de la imagen y el sonido, el apego a las reglas ortográficas y gramaticales y la relación entre imágenes, texto y narración. Otro apartado de la rúbrica es el diseño pedagógico, donde se revisa la precisión y actualización de la información presentada, la secuencia lógica del contenido abordado, la identificación de conceptos clave, el uso de recursos de soporte (gráficas, ilustraciones o ejemplos), la claridad y adecuación del lenguaje y el uso de recursos tipográficos y colores. Cada video se dividió en Introducción, donde se abordaron conceptos claves del tema; Desarrollo, donde se presentaron ejemplos de código de diferentes estructuras de programación en el lenguaje C; y Conclusión, donde se abordó un repaso del código presentado y se sugirieron al estudiante otros problemas que podían resolverse de manera similar. La tabla 1 presenta a detalle la información de cada video.

Tabla 1: Información de los videos educativos utilizados

\begin{tabular}{|c|c|c|c|c|c|}
\hline$\#$ & Título del video & Tema & Subtema & Objetivo & Duración \\
\hline 1 & $\begin{array}{l}\text { Estructuras de } \\
\text { control de } \\
\text { iteración parte } \\
\text { uno. }\end{array}$ & Ciclo do-while & $\begin{array}{l}\text { Ejemplo Negocio de } \\
\text { zapatería }\end{array}$ & $\begin{array}{l}\text { Ejemplificar el uso de do-while } \\
\text { controlado por centinela, uso de } \\
\text { acumuladores. }\end{array}$ & 18:35 min \\
\hline 2 & $\begin{array}{l}\text { Estructuras de } \\
\text { control de } \\
\text { iteración parte } \\
\text { dos. }\end{array}$ & Ciclo for y ciclo while & $\begin{array}{l}\text { Ejemplo administración } \\
\text { de información de } \\
\text { alumnos }\end{array}$ & $\begin{array}{l}\text { Ejemplificar el uso del del ciclo for } \\
\text { y while, marcar sus diferencias, } \\
\text { uso de contadores y } \\
\text { acumuladores. }\end{array}$ & 10:51 min \\
\hline 3 & $\begin{array}{l}\text { Estructuras de } \\
\text { control de } \\
\text { iteración parte } \\
\text { tres. }\end{array}$ & Ciclos anidados & $\begin{array}{l}\text { Ejemplo con pirámide } \\
\text { de números }\end{array}$ & $\begin{array}{l}\text { Ejemplificar el uso de ciclos } \\
\text { anidados, comportamiento de } \\
\text { contadores, en ciclo interno y ciclo } \\
\text { externo. }\end{array}$ & $7: 36 \mathrm{~min}$ \\
\hline 4 & $\begin{array}{l}\text { Arreglos parte } \\
\text { uno. }\end{array}$ & Vectores & Conceptos básicos & $\begin{array}{l}\text { Mostrar cómo declarar e inicializar } \\
\text { vectores int, float, char y su } \\
\text { representación visual. }\end{array}$ & 8:03 min \\
\hline 5 & $\begin{array}{l}\text { Arreglos parte } \\
\text { dos. }\end{array}$ & $\begin{array}{l}\text { Operaciones con } \\
\text { vectores paralelos }\end{array}$ & $\begin{array}{l}\text { Registro, consulta y } \\
\text { manipulación de datos } \\
\text { en vectores paralelos }\end{array}$ & $\begin{array}{l}\text { Ejemplificar el código de registro, } \\
\text { modificación, consulta, eliminación } \\
\text { y búsqueda en vectores paralelos. }\end{array}$ & 20:53 min. \\
\hline 6 & $\begin{array}{l}\text { Arreglos parte } \\
\text { tres. }\end{array}$ & $\begin{array}{l}\text { Ordenación de } \\
\text { vectores }\end{array}$ & $\begin{array}{l}\text { Algoritmo de } \\
\text { ordenación burbuja }\end{array}$ & $\begin{array}{l}\text { Ejemplificar la lógica del algoritmo } \\
\text { de ordenación burbuja y su } \\
\text { sintaxis. }\end{array}$ & 9:11 am \\
\hline 7 & $\begin{array}{l}\text { Arreglos parte } \\
\text { cuatro. }\end{array}$ & $\begin{array}{l}\text { Arreglos } \\
\text { bidimensionales }\end{array}$ & $\begin{array}{l}\text { Manipulación de } \\
\text { matrices }\end{array}$ & $\begin{array}{l}\text { Ejemplificar el registro y consulta } \\
\text { de matrices, así como la suma de } \\
\text { columnas, renglones y cálculo del } \\
\text { promedio de los datos } \\
\text { almacenados. }\end{array}$ & 19:03 min \\
\hline
\end{tabular}

\section{Estrategia didáctica}

Se diseñó e implementó una actividad de aprendizaje para la preparación de los estudiantes en su aplicación del tercer examen parcial de la unidad de aprendizaje de programación, con el objetivo de mejorar su aprendizaje e incrementar el porcentaje de aprobados. El examen abarca los temas de estructuras repetitivas, 
vectores, vectores paralelos y matrices. Se seleccionaron estos temas ya que se han identificado como de mayor dificultad de comprensión por parte de los estudiantes de otros ciclos escolares. La actividad de aprendizaje consistió en la entrega de cinco reportes semanales. La relación entre los reportes y los videos se muestra en la tabla 2. Se puede observar que los reportes uno y tres conllevaron dos videos, esto debido a la relación de los temas presentados y al tiempo destinado para abordar los contenidos en el curso. Las características del reporte indicadas fueron: 1) trabajo individual, 2) escrito en hoja blanca o reciclada, 3) portada con los datos del estudiante, 4) escritura a mano del código mostrado en el video, 5) explicación de las líneas del código, 6) fecha de entrega.

Tabla 2: Relación entre los reportes y los videos de la actividad.

\begin{tabular}{|l|l|}
\hline Reporte & Videos \\
\hline 1 & $\begin{array}{l}\text { Video uno: Estructuras de control de iteración parte uno: } \\
\text { Ciclo do-while } \\
\text { Video dos: Estructuras de control de iteración parte dos: } \\
\text { Ciclo for }\end{array}$ \\
\hline 2 & $\begin{array}{l}\text { Estructuras de control de iteración parte tres: Ciclos } \\
\text { anidados }\end{array}$ \\
\hline 3 & $\begin{array}{l}\text { Arreglos parte uno: Vectores } \\
\text { Arreglos parte dos: Operaciones con vectores }\end{array}$ \\
\hline 4 & Arreglos parte tres: Ordenación de vectores \\
\hline 5 & Arreglos parte cuatro: Arreglos bidimensionales \\
\hline
\end{tabular}

\section{Sujetos de estudio}

La muestra fue conformada por un total de 55 estudiantes de segundo semestre de ingeniería en la unidad de aprendizaje de Programación durante el ciclo escolar 2019-1 que abarca los meses de febrero a junio.

\section{Instrumentos}

Los videos educativos utilizados fueron creados con el software Camtasia, el cual facilitó la grabación de la información presentada en la computadora del docente, además de la grabación del audio y la imagen del mismo dando la explicación del tema. Estos materiales fueron dispuestos en un canal institucional de YouTube Ilamado REDIFIM (Recursos Didácticos Digitales de la FIM). Durante el periodo 2019-1, se diseñó y aplicó un instrumento para determinar la percepción de los estudiantes con respecto a los videos y la estrategia de aprendizaje implementada, mismo que estaba dividido en cuatro secciones. La primera sección contenía tres preguntas de opción múltiple y una pregunta abierta con las que se buscó conocer si los alumnos habían consultado los videos y en qué medida, así como el nivel de cumplimiento de la actividad de aprendizaje que se diseñó. La segunda sección se subdividió en siete preguntas con escala tipo Likert, cada una con una opción para describir libremente el porqué de la respuesta. Estas preguntas se enfocaron en determinar la experiencia en el uso de los videos, evaluando aspectos como duración, información presentada, la motivación que lograron en el estudiante, la coherencia en los temas, la actividad diseñada y el uso de videos como estrategia de enseñanza. La tercera sección constó de tres preguntas con opciones Sí y No, donde se evaluó el interés del estudiante por el uso de video de manera regular tanto en Programación como en otras unidades de aprendizaje. Finalmente, la cuarta sección constó de una pregunta abierta donde el estudiante podía dar comentarios adicionales sobre su experiencia con el uso de videos educativos.

\section{Evaluación de la estrategia}

Para evaluar el impacto de la estrategia de aprendizaje, se realizó un análisis de frecuencias para observar el comportamiento general de las respuestas de los estudiantes ante los diferentes cuestionamientos que se hicieron en la encuesta de percepción. Así mismo, se realizó un análisis cualitativo de las explicaciones dadas por los estudiantes para justificar sus respuestas. Por otro lado, se revisaron las calificaciones obtenidas por los estudiantes en la actividad didáctica y se compararon estos resultados con los de 413 alumnos del mismo docente en años anteriores con quienes no se aplicó esta estrategia. Para medir la confiabilidad del instrumento aplicado, en la sección que incluía escala Likert se realizó un cálculo del coeficiente Alfa de Cronbach, así como un análisis de estadística descriptiva utilizando el software PSPP (https://www.gnu.org/software/pspp/). 


\section{RESULTADOS}

En esta sección se presenta el análisis de frecuencia de los resultados obtenidos en la encuesta de percepción aplicada a los estudiantes sobre el uso de los videos educativos para el aprendizaje de la programación, un análisis cualitativo de las percepciones de los estudiantes, así como los resultados del análisis de calificaciones que obtuvieron los estudiantes.

\section{Análisis de frecuencia de la encuesta de percepción}

En la primera sección de la encuesta se aplicaron tres cuestionamientos para conocer si los alumnos habían consultado los videos y en qué medida, así como el nivel de cumplimiento de la actividad de aprendizaje que se diseñó. Se considera que, para una mejor apropiación de la información presentada en los videos y el logro del objetivo de la actividad didáctica, el alumno tendría que haber cumplido con la actividad en su totalidad, es decir, que entregará los cinco reportes, que viera los videos detenidamente y que consultara los videos por lo menos una vez o hasta que él se sintiera confiado de haber comprendido el tema explicado. Cabe aclarar que la encuesta fue anónima para evitar que el estudiante se sintiera supervisado en su respuesta y los resultados fueran mayormente objetivos.

Con respecto a las veces que el estudiante consultó cada video para una mejor comprensión, se obtuvo que un $50 \%$ de los estudiantes los consultó de una a cinco veces, el $25.9 \%$ una vez, el $13 \%$ de cinco a diez veces, el $7.4 \%$ más de 10 veces y el $3.7 \%$ ni una vez. El hecho de que existan estudiantes que no consultaron los videos se debe a que a la hora de plantear la actividad de aprendizaje se indicó que se trataba de una actividad adicional, por lo que no habría una penalización si no la realizaban. Para que la estrategia de aprendizaje fuera efectiva se requería que los estudiantes tomaran en serio la actividad y la realizaran correctamente. Para identificar si esto sucedió, se les preguntó si los videos fueron revisados detenidamente. Un $92.6 \%$ indicó haberlos revisado detenidamente, mientras que un $7.4 \%$ no lo hizo así.

Dentro de los reportes, la explicación de las líneas del código del programa, representa la parte principal de la actividad de aprendizaje, pues es donde el alumno tuvo que reflexionar sobre la explicación presentada en el video y plasmar la comprensión que alcanzó sobre el tema. Tratando de impulsar a los estudiantes para cumplir con la actividad, se les indicó que la entrega de los cinco reportes completos los haría acreedores a 10 puntos extras para su calificación en este examen. Cada reporte fue retomado en las sesiones presenciales de clase, lo cual además se aprovechó como aula invertida (Mason et al., 2013), y se proporcionaron nuevos ejemplos sobre el tema.

Con respecto a la cantidad de reportes entregados basados en la información proporcionada en los videos, existieron casos de estudiantes que no entregaron los cinco reportes solicitados. El mayor porcentaje se encuentra en la entrega del total de reportes (5) con un 36.4\%, seguido de la entrega de tres reportes con un $18.2 \%$, la entrega de cero reportes con visualización de los videos con un $12.7 \%$, la entrega de un reporte con el $10.9 \%$, la entrega de cero reportes y sin visualizaciones con un $9.1 \%$, la entrega de dos y cuatro reportes con $7.3 \%$ y $5.5 \%$, respectivamente. Se agregó una pregunta abierta para los estudiantes que no entregaron ningún reporte a fin de que indicaran el motivo. Las tres respuestas principales fueron que ya dominaban el tema, no tenían tiempo para hacerlo o se les había olvidado.

Con la información descrita anteriormente, se puede decir que menos de la mitad del grupo (36.40\%) cumplió con la actividad como se esperaba. La segunda sección de la encuesta se enfocó en determinar la experiencia del alumno en el uso de los videos, para lo cual respondieron los cuestionamientos con la escala tipo Likert: 5. Muy buena; 4. Buena; 3. Aceptable; 2. Deficiente; 1 . Muy deficiente. También se agregó una opción N/A para el caso de los estudiantes que no vieron los videos y que por tanto no tuvieron ninguna experiencia con respecto al uso. Fue en esta sección en donde se realizó el cálculo del coeficiente alfa de Cronbach. El valor alfa fue de 0.96, lo cual permite establecer que la sección del instrumento es confiable en términos de consistencia interna ya que el valor es cercano a uno.

El $10.9 \%$ de los estudiantes no vieron los videos por lo que las respuestas a los cuestionamientos de esta sección abarcan al $89.1 \%$ de los estudiantes que si los utilizaron. En cuanto a la duración de los videos, es decir, si el tiempo utilizado era suficiente tanto para mantener la atención del alumno como para transmitir la información, el $24.5 \%$ indicó que fue muy buena, el $53.1 \%$ que fue buena, el $20.4 \%$ que fue aceptable, el $2.0 \%$ respondió que fue deficiente. Cabe señalar que ningún estudiante señaló la opción de muy deficiente. Entre los comentarios hubo quienes expresaron que la duración fue lo suficiente para comprender el problema y hubo quienes dijeron que les hubiera gustado que la duración fuera menor.

En relación con la claridad de la información presentada en los videos, es decir, si el contenido presentado se explicaba de manera que se facilitara su comprensión, el $53.1 \%$ la consideró muy buena, el $26.5 \%$ buena, el $18.4 \%$ aceptable y el $2.0 \%$ la consideró deficiente. Las justificaciones fueron mayormente positivas ya que 
expresaban que la explicación era clara y los ejemplos sencillos. Otros comentarios fueron que la explicación era un tanto rápida o que faltaba mostrar el código completo en una sola toma para comprender mejor su estructura.

Sobre la motivación que el video generó en el alumno para invitarlo a cumplir con la actividad de aprendizaje y en si por el aprendizaje de la programación, el $51.1 \%$ otorgó una puntuación de muy buena ya que consideraban que el poder reforzar los temas y entenderlos era una motivación para seguir adelante con el curso, el $34.7 \%$ otorgó una puntuación de buena ya que los videos los ayudaban a realizar sus tareas, el $10.2 \%$ otorgó una puntuación de aceptable ya que sentían que su motivación no aumentó ni disminuyó con la visualización de los videos y el $4.0 \%$ otorgó una calificación de deficiente. Los comentarios fueron que les ayudaba a resolver sus dudas, a ganar puntos extras, a realizar mejor sus trabajos, a querer aprender programación. También se comentó que, debido a la falta de tiempo, a veces los estudiantes no podían ver los videos.

En cuanto a la coherencia en la sucesión de los temas, es decir, si lo abordado llevaba una secuencia que permitiera ir hilando los temas y relacionarlos para una mejor comprensión, el $65.4 \%$ de los estudiantes la consideró muy buena, el $22.4 \%$ otorgó puntuación de buena, el $10.2 \%$ consideró que la coherencia era regular y el $2.0 \%$ consideró que fue deficiente. Los comentarios fueron que los temas iban de acuerdo a las clases y que cada vez eran más complejos puesto que se requería conocer el tema anterior. Respecto a la opinión de los estudiantes sobre cómo el uso de los videos fomenta su curiosidad por aprender del tema, el $39.8 \%$ consideró que fue muy buena, el $49.9 \%$ que fue buena, el $6.3 \%$ que fue regular, seguida por el $2.0 \%$ que fue deficiente y el mismo porcentaje de $2.0 \%$ consideró que fue muy deficiente ya que no se fomenta la curiosidad por aprender del tema. Los comentarios expresaron que los motivaron a buscar más videos, a prepararse mejor para las clases y para obtener puntos extras.

Sobre la opinión de los alumnos respecto a la estrategia de aprendizaje utilizada, es decir, el hecho de que tuvieran que escribir un reporte explicando el código presentado en el video, el $55.6 \%$ consideró que es muy buena, el $35.6 \%$ consideró que fue buena, el $6.7 \%$ consideró que fue regular y el $2.1 \%$ consideró que fue deficiente. Los comentarios fueron que así se les quedaba mejor grabado el proceso, al escribir el código se entendía mejor, ayudaba a comprender la sintaxis y que cuando lo vas escribiendo la mente lo va analizando.

En relación a la puntuación otorgada a la estrategia de enseñanza con énfasis en el uso de videos, el 57.5.1\% de los alumnos otorgó una puntuación de muy buena ya que comentaron que esta estrategia fue práctica y se podían consultar los videos las veces que fueran necesarias, el 34.1\% otorgó una puntuación de buena pues consideraban que las dudas se podían aclarar mejor en clase, el $4.2 \%$ otorgó una puntuación de aceptable debido que esta estrategia podría ser un tanto confusa para algunos de los alumnos, el $4.2 \%$ otorgó una puntuación de deficiente ya que comentaban que en los videos había dudas específicas que no se podían resolver, y ningún estudiante otorgó una puntuación de muy deficiente. En los 55 casos evaluados, la media de respuestas estuvo en el rango de 3.56 a 4.02, es decir entre Aceptable y Buena, con una desviación estándar de 1.44 a 1.85 y una varianza de 2.07 a 3.42. La tabla 3 muestra la media, desviación estándar y varianza de los variables analizados anteriormente.

Tabla 3: Estadística descriptiva de los datos de la sección 2 del instrumento

\begin{tabular}{|l|c|c|c|c|}
\hline Variable & Número de casos & Media & Desv. Estd. & Varianza \\
\hline Duración & 55 & 3.56 & 1.44 & 2.07 \\
\hline Claridad & 55 & 3.84 & 1.57 & 2.47 \\
\hline Motivación & 55 & 3.85 & 1.57 & 2.46 \\
\hline Coherencia & 55 & 4.02 & 1.59 & 2.54 \\
\hline Curiosidad & 55 & 3.69 & 1.62 & 2.62 \\
\hline Estrategia de reporte & 55 & 3.64 & 1.85 & 3.42 \\
\hline Estrategia de video & 55 & 3.78 & 1.76 & 3.1 \\
\hline
\end{tabular}

En la sección 3 del instrumento se les cuestionó sobre la aplicación de la estrategia en todas las unidades y en otras asignaturas. En cuanto a sí le gustaría la utilización de videos como herramienta de apoyo en todas las unidades de la asignatura de Programación, el 53.7\% respondió que sí, mientras el $46.3 \%$ respondió que no. Entre sus razones se encontraron que no les gustó que fueran obligatorios y que consultar muchos videos es tedioso. La segunda pregunta estaba enfocada a si consideran factible que todo el curso se desarrolle con el uso de videos sin la intervención del docente, para lo que el $11.1 \%$ respondió que sí y el $88.9 \%$ respondió que no. Los comentarios fueron que prefieren mejor la clase presencial, se necesita un mentor, ya que no todos los alumnos son autodidactas. La última pregunta radicaba acerca de sí les gustaría que otras unidades 
de aprendizaje fueran apoyadas con el uso de videos, para lo cual el $85.2 \%$ respondió que sí y el $14.8 \%$ respondió que no. Los comentarios fueron que les serviría para repasar, que solamente lo ven útil para la asignatura de programación y que representan más opciones de aprendizaje. La Tabla 4 presenta las experiencias del estudiante con respecto al uso de la estrategia, preguntadas de manera abierta en la sección 4 del instrumento.

Tabla 4: Experiencia de los estudiantes con respecto a la estrategia

\begin{tabular}{|l|l|}
\hline \multicolumn{1}{|c|}{ Alumno } & \multicolumn{1}{c|}{ Experiencia } \\
\hline Alumno 1 & $\begin{array}{l}\text { Esperamos videos en materias como cálculo o estática explicados por los mismos docentes con los } \\
\text { que cursamos la materia. }\end{array}$ \\
\hline Alumno 2 & $\begin{array}{l}\text { Creo que los videos ayudan bastante para aprender de esta materia, pero también pienso que el } \\
\text { docente es esencial para el aprendizaje además de los videos. }\end{array}$ \\
\hline Alumno 3 & $\begin{array}{l}\text { Los videos fueron muy útiles para la comprensión de los últimos temas, así como de gran apoyo para } \\
\text { el proyecto final. }\end{array}$ \\
\hline Alumno 4 & $\begin{array}{l}\text { Todo el curso fue muy bueno, fue excelente que se agregaran los videos, pero siempre y cuando } \\
\text { también se vean los temas en clase, para aclarar dudas, pero teniendo como refuerzo los videos. }\end{array}$ \\
\hline Alumno 5 & Excelente método para hacer repasar a los estudiantes, muy recomendado. \\
\hline Alumno 6 & Más videos, con diferentes problemas para hacer otro programa. \\
\hline Alumno 7 & Creo que la enseñanza a partir de videos no motiva tanto como la presencial. \\
\hline Alumno 8 & $\begin{array}{l}\text { Falta mejorar un poco en la calidad del audio y tal vez poner al final del video una breve imagen con } \\
\text { el programa terminado. }\end{array}$ \\
\hline
\end{tabular}

\section{Análisis cualitativo}

Se realizó un análisis cualitativo con apoyo del software Nvivo para procesamiento y análisis de datos. El análisis se basa en los comentarios expresados por los estudiantes en las preguntas donde se les solicitó que explicaran la calificación otorgada a la variable evaluada. Los comentarios se clasificaron en comentarios positivos y negativos según lo expresado. También se identificaron comentarios que hacían sugerencias de mejora. La figura 1 muestra la cantidad de comentarios obtenidos. En color gris obscuro se encuentran los comentarios positivos, en color gris tenue los comentarios negativos y en gris claro las sugerencias de mejora. En total se obtuvieron 314 comentarios positivos, 126 comentarios negativos y 13 sugerencias de mejora. En todas las variables evaluadas los comentarios son mayormente positivos, principalmente la opción de contar con videos para otras materias (44). Solo la opción de llevar el curso sin apoyo del docente y solo con videos, fue donde lo comentarios negativos tuvieron la mayor proporción (46). La variable que menos comentarios negativos tuvo fue la Coherencia (2). Donde más sugerencias de mejora se identificaron fue en la opción de llevar el curso completo con apoyo de videos (6).

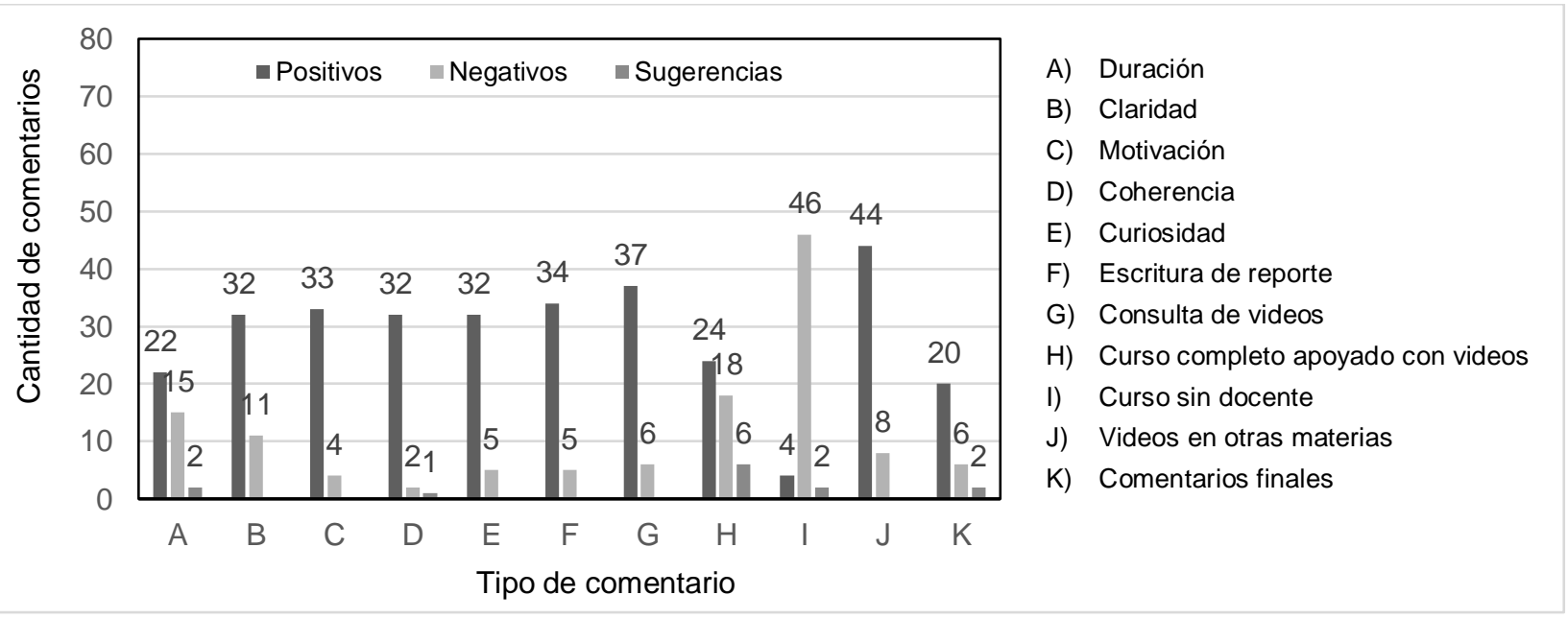

Fig. 1: Clasificación de comentarios hechos por los estudiantes (Elaboración propia).

\section{Comentarios positivos}

Los comentarios positivos reflejan de alguna forma que los estudiantes encontraron en la estrategia de aprendizaje una herramienta de apoyo adicional para la mejora en su comprensión de los temas de programación, reconocen a los videos educativos como un instrumento que tiene mayor disponibilidad que la que un maestro puede dar en el aula. Comprenden que la programación es difícil y que se requiere un esfuerzo 
extra para lograr su comprensión. Identifican a la escritura del código como un arma valiosa para el análisis de lo que transcriben y la apropiación de ese conocimiento. Hubo quienes no se quedaron solo con la información de la clase y los videos, sino que buscaron nuevos videos y otras fuentes para ampliar su conocimiento y ponerlo en práctica.

\section{Comentarios negativos}

Los comentarios negativos hacen referencia a las debilidades que identificaron en alguno de los videos o en conjunto por la estrategia. Destacan que la duración de algunos videos les pareció extensa, pues además de consultar el video requerían verlo múltiples veces para comprender mejor, además del reporte correspondiente. En algunos casos se consideró que la información se abordaba rápido, a pesar de que el video se podía detener en cualquier momento. Justifican el no haber cumplido con la actividad completa por falta de tiempo debido a actividades de otras materias o cuestiones personales. También se muestra que de alguna forma se sentían obligados a cumplir con la actividad, a pesar de que no había una penalización por no hacerlo. Existieron casos donde sintieron que el reporte realizado no aportó significativamente a su comprensión del tema, o el hecho de escribir a mano les pareció molesto.

\section{Sugerencias de mejora}

Los comentarios identificados como sugerencias de mejora hacen mención, de forma explícita, de algún aspecto a mejorar como es colocar en una sola pantalla el código completo, explicar más los detalles, solo considerar los videos como material de apoyo y no como algo obligatorio y utilizar los videos en los temas más complejos.

\section{Análisis de calificaciones}

Una manera de conocer si la estrategia didáctica implementada realmente tuvo un impacto en el aprovechamiento académico del estudiante fue al analizar sus calificaciones, específicamente del tercer examen parcial donde se evalúan los temas impartidos con la estrategia didáctica. Para dicho análisis, se consideraron los resultados obtenidos por los grupos del mismo docente desde el ciclo escolar 2015-1 al 2019-2. Cabe aclarar que son ciclos escolares semestrales los que maneja la UABC, cuyo ciclo escolar impar comprende los meses de enero a junio y el ciclo escolar par comprende de agosto a diciembre.

En el ciclo escolar par ingresan los estudiantes que obtuvieron mejores resultados en el examen de admisión a la UABC y el resto ingresa en el ciclo escolar impar. Dada esta situación y que la asignatura de interés para este estudio corresponde al segundo semestre, las generaciones de alumnos del período impar presentan mejores promedios en esta materia. La tabla 5 muestra, a manera de concentrado por cada periodo, la cantidad de alumnos que presentaron el tercer parcial, el número de aprobados $(A)$ y reprobados $(R)$ en el tercer parcial, el porcentaje de aprobados y reprobados en el tercer parcial y el promedio final del grupo en su evaluación global.

Tabla 5: Resultados del tercer parcial de Programación de 2015 a 2019

\begin{tabular}{|c|c|c|c|c|c|c|}
\hline Periodo & Alumnos & A & R & $\% A$ & $\% R$ & Promedio final global \\
\hline $2015-1$ & 43 & 23 & 20 & $5,348,837$ & $4,651,163$ & 72,105 \\
\hline $2015-2$ & 24 & 10 & 14 & $4,166,667$ & $5,833,333$ & 73,18 \\
\hline $2016-1$ & 61 & 42 & 19 & $6,885,246$ & $3,114,754$ & 83,11 \\
\hline $2016-2$ & 22 & 8 & 14 & $3,636,364$ & $6,363,636$ & 77,815 \\
\hline $2017-1$ & 46 & 20 & 26 & $4,347,826$ & $5,652,174$ & 74,96 \\
\hline $2017-2$ & 40 & 17 & 23 & 42,5 & 57,5 & 63,785 \\
\hline $2018-1$ & 60 & 34 & 26 & $5,666,667$ & $4,333,333$ & 77,935 \\
\hline $2018-2$ & 45 & 18 & 27 & 40 & 60 & 67,54 \\
\hline $2019-1$ & 55 & 38 & 17 & $6,909,091$ & $3,090,909$ & 74,42 \\
\hline $2019-2$ & 17 & 12 & 5 & $7,058,824$ & $2,941,176$ & 72.33 \\
\hline
\end{tabular}

Para interpretar la tabla 5, es necesario diferenciar entre los estudiantes del ciclo escolar par y los estudiantes del ciclo escolar impar. Para esto, la figura 2 muestra la gráfica comparativa entre el porcentaje de aprobados y el porcentaje de no aprobados en el tercer parcial, desde el periodo 2015-1 al periodo 2019-1. La figura 2 
muestra un comparativo entre los estudiantes del ciclo escolar impar. Se puede observar que en el periodo 2019-1 y 2016-1 se presentó el mayor porcentaje de aprobación, con 69.09\% y 68.85\% respectivamente; no obstante, es de resaltar que el promedio de aprobación en el resto de los ciclos escolares impares era de $55.62 \%$, observándose un incremento de $13.47 \%$ en la aprobación en 2019-1, ciclo donde se implementó la estrategia.

La figura 3 muestra el porcentaje de aprobados y el porcentaje de no aprobados, ordenados de menor a mayor periodo considerando tanto los ciclos escolares pares como impares. Se observa un comportamiento de zig zag en ambas líneas, donde el porcentaje de aprobados incrementa en los ciclos escolares impares y el porcentaje de no aprobados incrementa en los ciclos escolares pares. Además, en el ciclo escolar 2019-2 también se observa un incremento en el porcentaje de aprobados. Cabe aclarar que en dicho semestre se continuó con la estrategia, aunque no se encuestó a los estudiantes porque aún se estaban procesando los resultados del estudio.

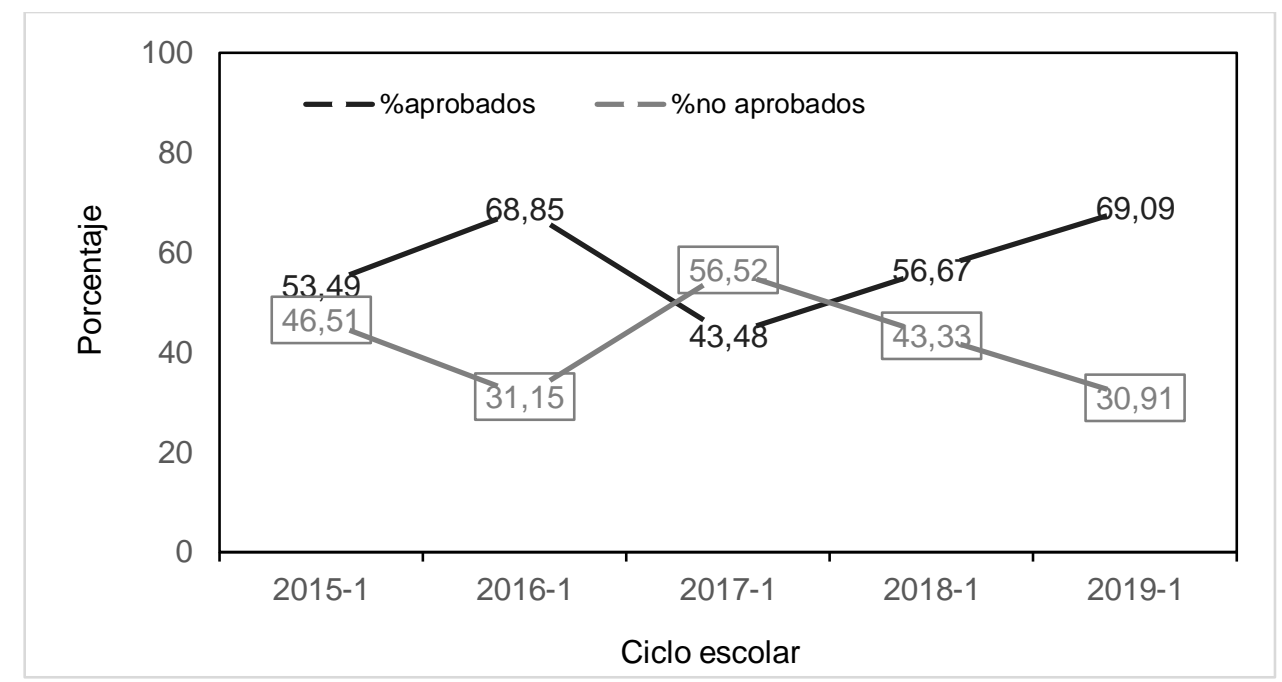

Fig. 2: Aprobados y no aprobados en el ciclo escolar impar desde 2015-1 hasta 2019-1 en el tercer parcial de Programación

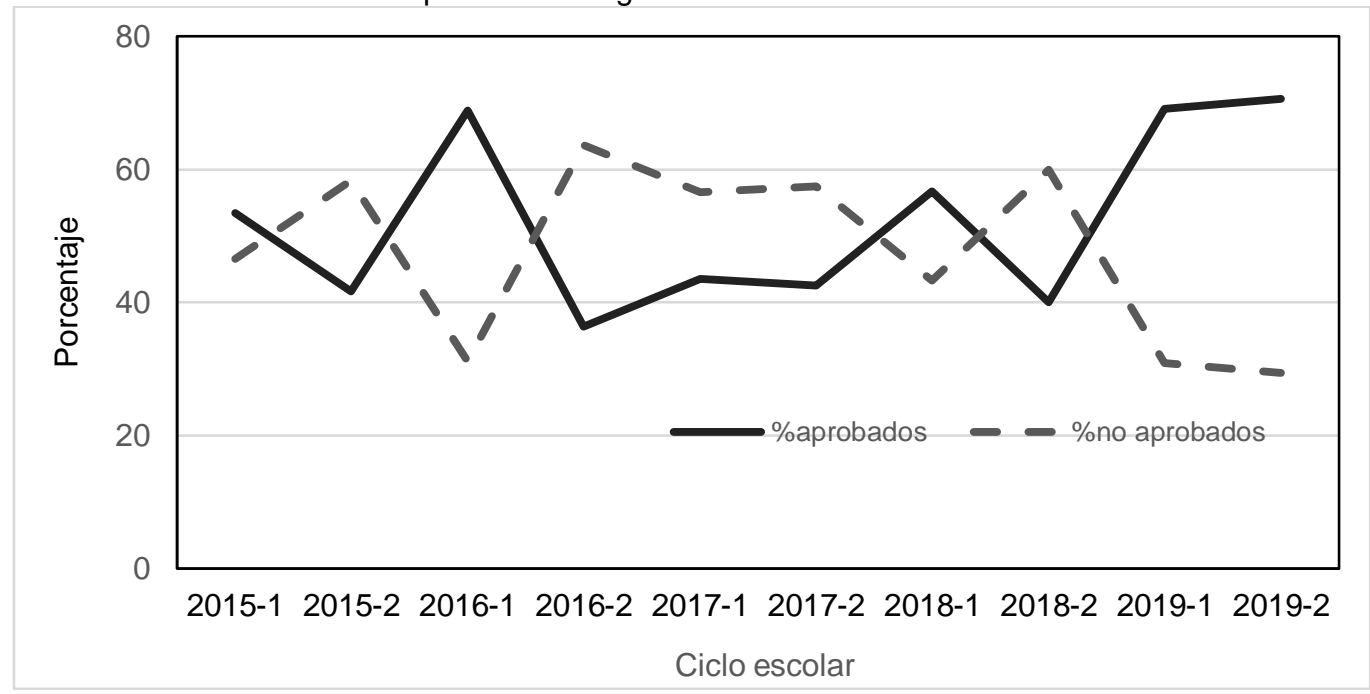

Fig. 3: Aprobados y no aprobados en el tercer parcial de Programación desde 2015 a 2019

La figura 4 muestra el comportamiento del promedio global de los alumnos desde 2015 a 2019 . Se observa nuevamente la tendencia de zig zag, donde el promedio es mayor en los ciclos escolares impares. También se observa que el mayor promedio se presentó en el 2016-1 y en el 2018-1, semestres en los que no se aplicó la estrategia, mientras que en el 2019-1 el promedio fue de 74.42 . 


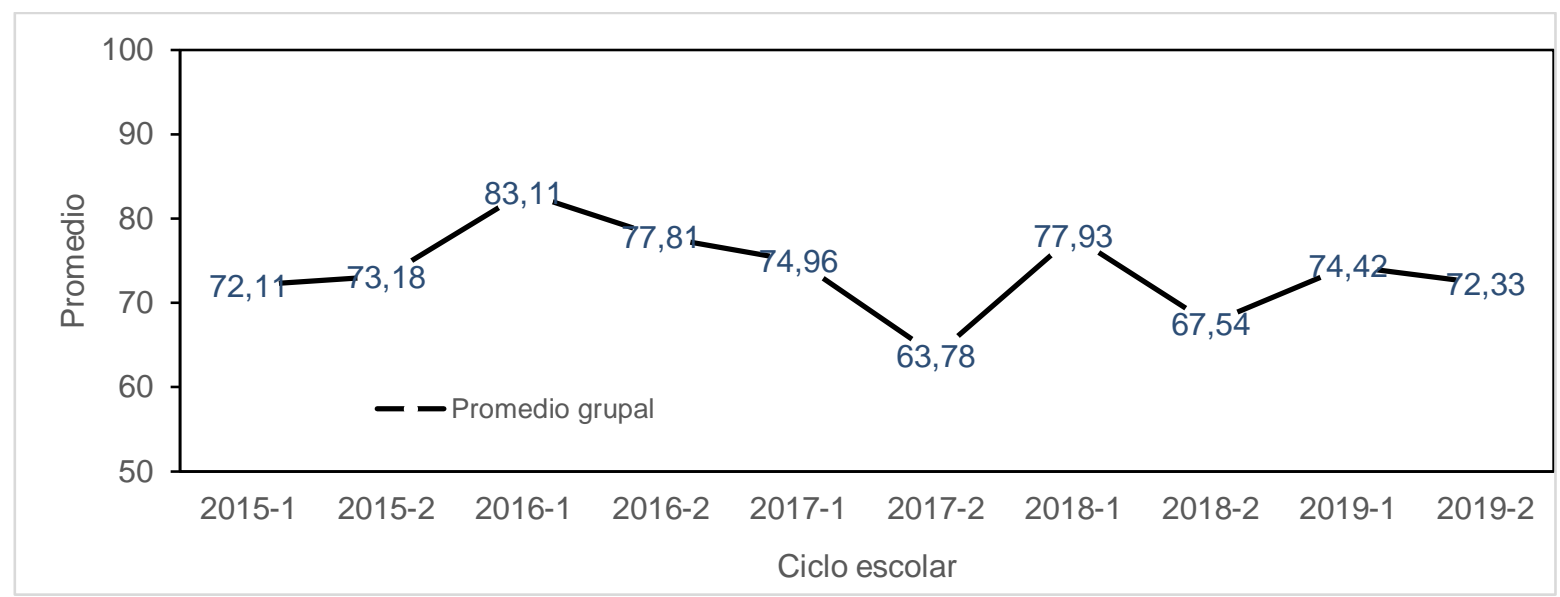

Fig. 4: Promedio final global en los grupos de Programación desde 2015 hasta 2019 (Elaboración propia).

\section{DISCUSIÓN}

La materia de programación en la formación de los ingenieros, favorece el pensamiento lógico y crítico para que adquieran las competencias básicas que les permitan desarrollar soluciones tecnologías para resolver problemas de ingeniería mediante el pensamiento computacional. Esta materia no tiene el objetivo de que cada estudiante se vuelva un programador profesional. Es por esto que se hacen esfuerzos por implementar estrategias que faciliten la comprensión de la lógica de la programación y en el caso particular de este trabajo, la estrategia del uso de videos educativos resulta beneficiosa por la facilidad que tienen los estudiantes de consultar Internet en sus celulares o computadoras personales, así como por el interés que tienen en el uso de las tecnologías de la información y comunicación (Fracchia et al., 2016). El análisis realizado ha permitido identificar las ventajas que ofrece el uso de los videos en el aprendizaje de la programación, así como los aspectos que se deben reforzar para lograr en mayor medida un impacto positivo en el uso de estos recursos. Velázquez y González (2017) aseguran que se tiene que buscar la motivación en el estudiante para mejorar su desempeño. En este sentido, la estrategia logró que solo el $36.40 \%$ de los estudiantes participantes encontraran la motivación que les permitiera cumplir con las actividades solicitadas en su totalidad, el resto lo hizo parcialmente. Aun así, se observó un incremento en el porcentaje de aprobación del tercer parcial en 2019-1 del $13.47 \%$.

La encuesta arrojó que para un $87.3 \%$ la claridad de los videos era aceptable, y en cuanto a la coherencia y duración de los mismos el porcentaje de aceptación por parte de los alumnos también estuvo por arriba del $80 \%$, lo que permite descartar que la falta de interés por completar la actividad se debió exclusivamente a la calidad de los recursos. En concordancia con que le mencionan Ann y Carr (2017) sobre los diferentes estilos de aprendizaje para diferentes alumnos, es imposible influir de manera positiva con una sola estrategia en el $100 \%$ de la población, puesto que cada individuo desarrolla un proceso diferenciado de aprendizaje, el cual atiende en cierta medida a sus propias convicciones.

Se logró constatar que el uso de este tipo de herramienta digital es un factor de motivación en el estudiante para aprender los temas de programación, ya que un $85.5 \%$ contestó de forma positiva a las preguntas sobre la motivación e interés que fomentaron los videos por aprender del tema. No obstante, acorde con BeltránPellicer, Giacomone y Burgos (2018) donde se sugiere que los docentes sean cuidadosos seleccionando y recomendando aquellos vídeos que mejor se adapten al alumno, estos expresaron que la intervención docente es fundamental para la enseñanza de la programación, puesto que en las preguntas abiertas mencionaron que es necesaria para aclarar dudas de manera inmediata.

A pesar de que la actividad realizada tuvo una buena aceptación por parte de los estudiantes (78.2\%), en la pregunta relacionada con tener más videos para apoyar el curso completo, solo el $53.70 \%$ contestó a favor de esta iniciativa, lo cual puede atribuirse a que el hecho de utilizar los videos como parte de una actividad didáctica implicaba un mayor tiempo de dedicación, puesto que tenía que verse el video y después atender la actividad. El tiempo de visualización de un video es el tiempo que el cerebro humano interactúa con el contenido, lo cual implica mayor compromiso cognitivo (Moreno, 2009). En este sentido, un 50\% indicó que tenía que ver los videos de dos a cinco veces.

Resultó importante saber si para el alumno era factible aprender programación sin la intervención del docente y solo con el apoyo de videos educativos. El $88.90 \%$ de los estudiantes no estuvo de acuerdo con que el curso completo de programación se desarrolle solo con videos educativos sin la intervención explicativa y 
presencial del docente, puesto que como indican Tapia et al., (2020) los videos apoyan en la motivación de los estudiantes para aprender; sin embargo, tal como establece Trujillo (2011) la verdadera utilidad de los recursos no se encuentra solo en su uso, sino también en el sentido pedagógico que se logra a través de la guía del docente. Como señala García (2014), el video carece de ventajas didácticas si no viene acompañado con una guía que oriente al aprendiz y al docente, en las distintas fases de los procesos de enseñanza y de aprendizaje.

Considerar estos resultados con lo mencionado por Vázquez et al., (2013) sobre la importancia del diseño instruccional en un curso a distancia, invita a reflexionar sobre la posibilidad de introducir a los estudiantes a cursos en línea, donde la cátedra docente disminuye significativamente. Se observó que los estudiantes universitarios, a pesar de ser nativos digitales y de la capacidad de autoaprendizaje que desarrollan actualmente en las aulas, visualizan a la intervención docente como parte fundamental para lograr sus procesos de aprendizaje en el área de programación y consideran a la tecnología como un acompañamiento que no reemplaza a la intervención humana. No se reconocen como estudiantes autodidactas. Tal como lo explica Hubalovsky (2015), la falta de retroalimentación inmediata entre estudiantes y maestros en un curso a distancia, trae como consecuencia menos posibilidades para el desarrollo del conocimiento teórico y habilidades prácticas de los estudiantes.

En el análisis cualitativo se identificaron comentarios positivos y negativos hacia la estrategia implementada, $69.30 \%$ fueron positivos, $27.80 \%$ negativos y $2.9 \%$ sugerencias de mejora. En algunos casos los comentarios entran en contradicción, puesto que hay quienes dicen que el video era claro, la estrategia buena, el aprendizaje logrado, y hay quienes dicen que no fue del todo claro, que la estrategia no fue de ayuda o que aprendieron más con lo explicado en clase. No obstante, este análisis reveló aspectos a considerar en la implementación de la actividad para un mayor impacto. En cuanto a los videos se refiere es importante cuidar la duración y el detalle de la información presentada, es mejor hacer varios videos cortos y bien explicados, que un video largo que puede llegar a abrumar y perder la atención del usuario.

En el trabajo de Xie et al., (2017) se analizó la relación entre el tiempo de un video y el tiempo que se mantiene el usuario en este, y cuando la duración es mayor a 10 minutos, disminuye la probabilidad de que se finalice la consulta del video. Para el estudiante es mejor la explicación en vivo del docente para poder hacerle preguntas, pero el video es de utilidad ya que lo puede consultar en cualquier momento y repetirlo o detenerlo para entender mejor. En este estudio los estudiantes expresaron las razones por las cuales consultar videos educativos, las cuales coinciden con Borges y Silveira (2019), quienes exponen que estas razones son: mejorar el aprendizaje, acceder a clases perdidas, aprender para exámenes y pruebas previas, estudiar con anticipación para la clase, entre otros. Resulta conveniente acompañar el curso completo de videos preseleccionados y determinar los temas en los que el reporte escrito puede apoyar más. De esta manera el estudiante tiene acceso a mayor cantidad de ejemplos y al consultarlos en YouTube, puede encontrarse con códigos de otros autores, hacer sus propias búsquedas, explorar, probar y aumentar su práctica. Una gran cantidad de recursos educativos gratuitos están disponibles en la web, a los cuales el estudiante puede tener acceso, pero es crucial que el alumno tenga la capacidad de hacer búsquedas eficientes para que los pueda aprovechar mejor en sus procesos de aprendizaje.

Los videos de YouTube pueden generar un impacto positivo en las calificaciones de los estudiantes. Los videos educativos tienen potencial como apoyo al aprendizaje no solo para la materia de programación, si no para otras materias donde servirían de material para asesorías y para homogeneizar formas de enseñanza entre diferentes docentes. Es importante combinarlos con otras estrategias de enseñanza, para hacer más dinámico el proceso de aprendizaje, y que no se vuelvan un mecanismo de distracción, pues hay que reconocer que al estar dispuestos en YouTube el alumno tiene acceso a otro tipo de material que lo distrae de su objetivo de aprendizaje (Flores et al., 2017).

En cuanto a la revisión de los porcentajes de aprobación y reprobación que obtuvieron los estudiantes en el tercer parcial de Programación, así como la comparación de los resultados del tercer parcial que históricamente han tenido los estudiantes del docente que aplicó la estrategia, el promedio del porcentaje de aprobados de 2015-1 a 2019-1 fue de 58.31\%. El periodo 2019-1 presentó un porcentaje de aprobados de 69.09\%, es decir, 10.78 puntos superior al promedio. No obstante, para alcanzar una conclusión que no sólo incluyera el resultado de un examen, también se revisó el resultado final de los estudiantes en la unidad de aprendizaje, contemplando todas las unidades temáticas y no solo aquellas en la que se implementó la estrategia de videos educativos.

La media aritmética de calificación final de los periodos impar desde 2015-1 a 2019-1 fue de 76.50, siendo esta calificación por arriba del promedio obtenido en 2019-1, que fue de 74.42. Con esta aclaración, es posible establecer que, si bien el apoyo de los videos educativos combinados con una estrategia de enseñanza favoreció los resultados parciales de los estudiantes, el resultado global no se vio totalmente influido, lo cual es esperado ya que la estrategia se focalizó en temáticas seleccionadas. 


\section{CONCLUSIONES}

El uso de los videos educativos reportados en este trabajo para apoyar el aprendizaje de la programación en ingeniería fue considerado aceptable por los estudiantes, los reconocen como una herramienta valiosa de apoyo, aunque para ellos es importante contar con la guía y asesoría del docente, de otra manera les resulta complicado lograr las competencias del curso. En el estudio realizado solo se consideraron los temas que se evalúan en el tercer examen parcial de la unidad de aprendizaje, lo que dio como resultado una mejora solamente en las calificaciones de dicho examen. Se considera que, para tener un impacto de mayor alcance, es necesario realizar actividades similares a lo largo del curso, donde se proporcionen al estudiante este tipo de recursos como complemento en cada uno de los temas de la materia, siempre enmarcados dentro de una secuencia didáctica en la que se permita la interacción entre el material, el docente, el estudiante y los objetivos de aprendizaje. Así mismo, los resultados muestran que los estudiantes esperan contar con este tipo de recursos en otras materias y no solo de programación en C, por lo que pueden ser útiles en otras áreas del conocimiento. Su implementación en modalidades de aprendizaje hibridas y la consulta a egresados sobre la importancia de la programación en su ámbito laboral, pueden ser variables a estudiar para un trabajo futuro.

\section{REFERENCIAS}

Ann, D., y Carr, M., Learning styles theory fails to explain learning and achievement: Recommendations for alternative approaches, doi: https://doi.org/10.1016/j.paid.2017.04.050, Personality and Individual Differences, 116, 410-416 (2017)

Armoni, M., Meerbaum-Salant, O., y Ben-Ari, M., From scratch to "real" programming, doi: https://doi.org/10.1145/2677087, ACM Transactions on Computing Education. 14(4), 1-15 (2015)

Bashir, G., y Hoque, A., An effective learning and teaching model for programming languages, https://doi.org/10.1007/s40692-016-0073-2, Journal Computer and Education. 3, 413-437 (2016)

Beltrán-Pellicer, P., Giacomone, B., y Burgos, M., Los vídeos educativos en línea desde las didácticas específicas: el caso de las matemáticas, DOI: 10.1080/11356405.2018.1524651, Culture and Education, 30(4), 633-662 (2018)

Bosse, Y., y Gerosa, M., Why is Programming so difficult to learn? Patterns of difficulties related to programming learning mid-stage, doi: 10.1145/3011286.3011301, Association for Computing Machinery, 41(6), 1-6 (2017)

Borges, P. R. S., y Silveira, I. F., Adding and segmenting educational videos: experiences of teacher users in an educational portal, doi: 10.1109/ACCESS.2019.2924946, IEEE Access, 7, 87996-88011 (2019)

Cano, E. The rubrics as an instrument for assessing competencies in higher education: use or abuse? Profesorado, Revista de Currículum y Formación del Profesorado, 19 (2), 1-16 (2015)

De la Fuente, D., Hernández, M., y Pra, I., Vídeo educativo y rendimiento académico en la enseñanza superior a distancia, doi: http://dx.doi.org/10.5944/ried.21.1.18326, Revista Iberoamericana de Educación a Distancia, 21(1), 323$341(2018)$

Derus, S.R., y Ali, A.Z., Development of hardware-interfacing learning kit for novice learning programming. doi:10.7763/IJIET.2016.V6.767, Journal of Information and Education Technology, 6, 647-651 (2016)

Eslava, M., López, O., Lloclla, H., y Vidaurre W., Videos educativos como estrategia tecnológica en el desempeño profesional de docentes de secundaria, Revista Venezolana de Gerencia, 23(84), (2018)

Flores, G.A., Chancusig, J.C., y otros tres autores, La influencia de las redes sociales en los estudiantes universitarios, Revista Boletín Redipe, 6(4), 56-5 (2017)

Fracchia, C., Kogan, P., y Amaro, S., Competir + motivar + hornero = aprender programación, Revista lberoamericana de Tecnología en Educación y Educación en Tecnología, (18), 19-29 (2016)

Gago, A., y Vico, M., Generación de material audiovisual para el aprendizaje basado en tareas o proyectos: cómo mejorar el aprendizaje con el apoyo de videos guía o explicativos, Revista Internacional de Educación y Aprendizaje, 1(2), 49-64 (2013)

García, M., Uso instruccional del vídeo didáctico, Revista de investigación, 38(81), 43-68 (2014)

Hubalovsky, S., Remote contact learning as method of teaching of algorithm development and programming in distance study, doi: 10.1016/j.sbspro.2015.04.667, Procedia - Social and Behavioral Sciences, 191(2), 1958-1963 (2015)

Kanoh, H., The learning motivation and understanding towards programming education of elementary school students in Japan, doi: 10.18178/ijiet.2018.8.12.1153, International Journal of Information and Education Technology, 8(12), 855860 (2018)

Khaleel, F., Shaari, N., y Wook, T., An empirical study on gamification for learning programming language website, J. Teknol., 81(2), 151-162 (2019)

Lozano, A., Zárate, J., y Laven, M., Uso de recursos educativos en línea en el nivel medio superior. Desarrollo de competencias didácticas del docente, doi: 10.25009/cpue.v0i26.2539, Revista de Investigación Educativa, 1(26), 114$135(2018)$ 
Mason, G. S., Shuman, T. R., y Cook, K. E., Comparing the effectiveness of an inverted classroom to a traditional classroom in an upper-division engineering course, doi: 10.1109/TE.2013.2249066, IEEE Transactions on Education, 56(4), 430-435 (2013)

Matthews, R., Hin, H.S., y Choo, K.A., Comparative study of self-test questions and self-assessment object for introductory programming lessons, doi: https://doi.org/10.1016/j.sbspro.2015.01.466, Procedia - Social and Behavioral Sciences, 176, 236-242 (2015)

Moreno, R., Constructing knowledge with an agent-based instructional program: a comparison of cooperative and individual meaning making, doi: https://doi.org/10.1016/j.learninstruc.2009.02.018, Learning and Instruction, 19(5), 433444 (2009)

Odo, C., y Odo, A., Effect of simulation on students' interest in programming language in secondary schools in Enugu education zone of Nigeria, doi: 10.7763/IJIET.2016.V6.73, International Journal of Information and Education Technology, 6, 477-480 (2016)

Ríos, J., Uso didáctico del vídeo. Temas para la Educación, Revista Digital para Profesionales de la Enseñanza, (13) 1-5 (2011)

Solano, I., y Sánchez, V., Aprendiendo en cualquier lugar: podcast educativo, Pixel-Bit Revista de Medios y Educación, (36), 125-139 (2010)

Tapia, J., Sánchez, A., y Vidal, C., Estilos de aprendizaje e intención de uso de videos académicos de youtube en el contexto universitario chileno, doi: 10.4067/S0718-50062020000100003, Formación universitaria, 13(1), 3-12 (2020)

Topalli, D., y Cagiltay, N.E., Improving programming skills in engineering education through problem-based game projects with scratch, doi: https://doi.org/10.1016/j.compedu.2018.01.011, Computers \& Education, 120, 64-74 (2018)

Trujillo, J., Comunicación, innovación, educación y gestión del conocimiento en torno al uso del podcast en la educación superior, doi: http://dx.doi.org/10.7238/rusc.v8i2.1047, Revista de Universidad y Sociedad del Conocimiento, 8(2), 61-76 (2011)

Vázquez, E., Vite, S., y Contreras, S., Diseño instruccional en la educación a distancia: la importancia y contribución del tecnopedagogo, Apertura, 4(2), 106-115 (2013)

Velázquez, Y., y González, M., Factores asociados a la permanencia de estudiantes universitarios: caso UAMM-UAT, https://doi.org/10.1016/j.resu.2017.11.003, Revista de la Educación Superior, 46(184), 117-138 (2017)

Xie, T., Zheng, Q., Zhang, W., y Qu, H., Modeling and predicting the active video-viewing time in a large-scale elearning system, doi: 10.1109/ACCESS.2017.2717858, IEEE Access, 5, 11490-11504 (2017) 